\title{
Die Telefonseelsorge Österreich - Telefonisches und digitales Krisenmanagement: Entwicklungslinien und Trends
}

\author{
Petra Schadenhofer (D) - Harald Stummer (D) - Lina M. Curth
}

Angenommen: 15. November 2021 / Online publiziert: 10. Dezember 2021

(c) Der/die Autor(en) 2021

\begin{abstract}
Zusammenfassung Der Notruf 142 der Telefonseelsorge (TS) spielt eine bedeutende Rolle im Feld der Krisenhotlines. Alle in Österreich lebenden Personen erhalten bei Bedarf eine niederschwellig zugängliche, kostenlose und vertrauliche Krisenintervention. Das rund um die Uhr erreichbare Angebot der TS umfasst Beratungs- und Entlastungsgespräche, stabilisierende Begleitung in schwierigen Lebenssituationen, Klärung des psychosozialen Bedarfes und Krisenintervention mit dem speziellen Schwerpunkt Suizidprävention (24/7).

Eine aktuelle Studie ( $n=374)$ während der Sars-CoV2Pandemie zeigte die Relevanz der TS und die Themenverteilungen der Beratungen auf - nämlich Einsamkeit, psychisches Wohlbefinden, Berufsleben, Abhängigkeitserkrankungen, Sinnempfinden, Suizid und Gewalt. Im Jahr 2020 ergaben sich in Österreich etwa 153.000 Kontakte, davon etwa $96 \%$ per Telefon und je etwa zwei Prozent per E-Mail und Chat (dies entspricht besonders in der Chatberatung einer Zunahme um $300 \%$, wobei der Chat noch nicht ganztägig besetzt ist).
\end{abstract}

P. Schadenhofer $(\bowtie)$

Diözese St. Pölten, Telefonseelsorge Niederösterreich, Klostergasse 15, 3100 St. Pölten, Österreich

p.schadenhofer@kirche.at

\section{H. Stummer}

Medizinische Informatik und Technik, Department für Public Health, Versorgungsforschung und Health Technology Assessment, Institut für Management und Ökonomie im Gesundheitswesen, UMIT -

Private Universität für Gesundheitswissenschaften,

Eduard-Wallnöfer-Zentrum 1, 6060 Hall in Tirol, Österreich

\section{M. Curth}

Diözese Linz, Telefonseelsorge Oberösterreich, Schulstraße 4, 4040 Linz, Österreich
Der vorliegende Artikel beleuchtet (a) die Bedeutung der Niederschwelligkeit, Kostenfreiheit, Vertraulichkeit und der regionalen Vernetzung der Telefonseelsorge und (b) die Entwicklungstrends der Beratung durch die und in den digitalen Medien. Dabei zeigen sich Veränderungen hinsichtlich der Altersgruppen der Nutzer_innen und Beratungsthemen je nach verwendetem Medium.

Schlüsselwörter Telefonseelsorge - Notruf · Onlineberatung $\cdot$ Krisenmanagement . Vertraulichkeit · Niederschwelligkeit

Telephone emergency service in Austria-crisis management by phone and digital: development course and trends

Summary The Telephone Emergency Service (TES, in Austria: the phone number 142) plays an important role regarding immediate counseling in crisis. Everyone living in Austria has the possibility to get a lowthreshold, free-of-charge and confidential crisis intervention. TES gives support by means of counseling, relief talks and stabilizing backing in difficult life situations, e.g. through evaluating the psychosocial demand. One special focus is on the $24 / 7$ suicide prevention availability.

There is little research done about the TES, however a recent study reveals its relevance and the recent trends: psychological and social well-being, professional life, drug addiction, the meaning of life and meaning-making, suicide and violence. In 2020, in Austria 153,000 contacts reached TES-roughly 96\% contacted the TES by telephone, $2 \%$ via e-mail and also $2 \%$ using an internet chat function, whereby the chat is staffed not yet 24/7. But, the number of chat contacts tripled within one year. As little research has been done on TES, the present paper draws on (a) the 
necessity of low-barrier contacts, the free-of-charge service, the confidentiality and the regional cross-linkages of the TES. One special focus are (b) the trends because of using new media. There, significant differences in age-cohorts, but also in topics for counseling could be found.

Keywords Telephone Emergency Service • Emergency Call · Online Counseling · Crisis Intervention · Confidentiality · Low-threshold counseling

\section{Die Telefonseelsorge - Notruf 142}

Die Telefonseelsorge spielt eine wichtige Rolle im Bereich der telefonischen (Rek und Dinger 2016) und digitalen (Wenzel 2008) Krisenintervention mit einem besonderen Schwerpunkt auf Suizidprävention. Das Beratungsangebot dieser Krisenhotline bietet vor allem Menschen, die sich in einer schwierigen Lebenssituation oder Krise befinden, ein offenes Ohr, Entlastung, Unterstützung - unabhängig von deren Alter, Geschlecht, Religion und sozialer Herkunft.

In Österreich ist die Telefonseelsorge unter der amtlichen Notrufnummer 142 rund um die Uhr (24/7), gebührenfrei, vertraulich und niederschwellig erreichbar. Sie arbeitet mehrheitlich mit ehrenamtlich tätigen, speziell geschulten Mitarbeiter_innen und ist bundesländerweise organisiert sowie regional vernetzt. Im Jahr 2012 wurde die Telefonseelsorge in Österreich durch das Angebot der Mailberatung und 2016 durch die Chatberatung - zunächst ausschließlich mit Terminvereinbarung über die TS-Homepage erweitert.

Die Telefonseelsorge ist zudem auch international organisiert. Die „International Federation of Telephone Emergency Services (IFOTES)“ wurde 1967 als Non-Profit-Organisation mit Sitz in der Schweiz gegründet und auch in Udine (Italien) registriert. Die Gründung erfolgte mit dem Ziel, Menschen in psychischen Krisen, Einsamkeit oder mit Suizidabsichten unverzüglich zugängliche, emotionale Unterstützung zur Verfügung zu stellen. 31 Organisationen aus 24 Ländern haben sich IFOTES angeschlossen eine davon ist die Telefonseelsorge Österreich. IFOTES wird von 338 Telefonzentralen, von über 20.000 geschulten ehrenamtlichen Mitarbeiter_innen und von über 800 hauptamtlich Angestellten getragen (IFOTES 2021).

In der 1966 in Österreich gegründeten Telefonseelsorge arbeiten heute in neun Telefonzentralen 886 professionell ausgebildete ehrenamtliche Mitarbeiter_innen und 31 hauptamtlich Angestellte. Das Durchschnittsalter der Berater_innen beträgt im Mittel 58 Jahre $(M=57,65, S D=12,43$; Humer et al. 2021). Pro Telefonzentrale sind oft mehrere Telefonleitungen besetzt. Regelmäßige fachlich spezifische Fortbildungen, Intervisions- und Supervisionsangebote sowie bundesländerübergreifender Informationsaustausch und Support bei technischen Herausforderungen sind als Qualitätsmerkmale der TS Österreich verankert. Zudem stehen den Berater_innen - neben Beratungstools - rund um die Uhr interne Ansprechpersonen (im Sinne von Bereitschaftsdiensten hauptamtlicher Mitarbeiter_innen) zum zeitnahen fachlichen Austausch zur Verfügung.

Hinsichtlich des Krisenmanagements ermöglicht die Telefonseelsorge häufig eine Verbindung zur psychotherapeutischen Face-to-Face-Behandlung. Etwa kann im Beratungsverlauf dazu ermutigt werden, Psychotherapeut_innen zu konsultieren. So ergab sich seit dem Jahr 2020 in Oberösterreich eine Kooperation mit niedergelassenen Psychotherapeut_innen: Anrufende können über die Telefonseelsorge fünf Stunden Psychotherapie kostenfrei erhalten, welche von der Österreichischen Gesundheitskasse (ÖGK) finanziert werden. Ergänzend dazu kann im psychotherapeutischen Prozess auf in Anspruch genommene Onlineoder Telefonberatungen Bezug genommen werden. Dieser Brückenschlag wird besonders dadurch unterstützt, dass das Angebot der Telefonseelsorge von den Kontaktsuchenden äußerst niederschwellig in Anspruch genommen werden kann (siehe z. B. Knatz und Dodier 2003, S. 16f.).

\section{Telefonisches und digitales Krisenmanagement}

Nach Caplan (1964) und Cullberg (1978) wird „Krise“ als „Verlust des seelischen Gleichgewichts“ beschrieben, und zwar insofern, indem

ein Mensch verspürt, wenn er mit Ereignissen und Lebensumständen konfrontiert wird, die er im Augenblick nicht bewältigen kann, weil sie von der Art und vom Ausmaß her seine durch frühere Erfahrungen erworbenen Fähigkeiten und erprobten Hilfsmittel zur Erreichung wichtiger Lebensziele oder zur Bewältigung seiner Lebenssituation überfordern (zit. nach Sonnek et al. 2012, S. 15).

Bei der Bewältigung von Krisen sind interne und externe Perspektiven relevant. So beschreiben aktuelle Studien einen Zusammenhang interner und externer Perspektiven im Krisenmanagement (Bundy et al. 2017; Mikušová und Horváthová 2019; Tagarev und Ratchev 2020). Dabei fokussiert die interne Krisenperspektive die Krisenbewältigung: Nach James et al. (2011) ist die Fähigkeit, Herausforderungen und Krisen konstruktiv zu bewältigen, unter anderem besonders darauf begründet, dass Verständnismöglichkeiten betrachtet und gegenübergestellt werden, bzw. dass dadurch schlussendlich neue Erfahrungen und Bewältigungsstrategien generiert werden (James et al. 2011). In diesem Sinne bestätigen zum Beispiel Madsen und Desai (2010) einen positiven Einfluss von Krisen auf die Lernfähigkeit sowie auf die Motivation der Betroffenen. Dies wiederum erhöht die Wahrscheinlichkeit einer umfassenden Suche nach der 
Ursache und adäquaten Lösungsansätzen (Madsen und Desai 2010). Dabei unterstützt die Beratung mittels eines transparenten, interaktiven Dialogs, durch das gemeinsame Recherchieren relevanter Informationen (du Plessis 2018) und das Zusammenführen interner und externer Perspektiven. Ergänzend dazu steht die Wahrnehmung von Krisen im Fokus externer Krisenperspektiven. Studien beschäftigten sich bei der Untersuchung „externer Krisenperspektiven“ vorwiegend damit, wie Krisen wahrgenommen werden, wie auf sie reagiert wird und wie Gesellschaft, Organisationen, Beratungseinrichtungen das Krisenempfinden beeinflussen (Bundy et al. 2017).

In der täglichen Arbeit der TS ist das Ziel der Krisenintervention, im Rahmen eines ersten Gesprächs (unabhängig vom Medium) einen guten, vertrauensvollen Kontakt herzustellen sowie die aktuelle Problemlage bzw. Situation zu erfassen (Stein 2015). Gerade in der praktischen Erfahrung zeigt sich, dass diese wichtige Basis der Krisenintervention einen positiven Effekt hinsichtlich der Bewältigung der eigenen Lebenssituation in allen drei Formaten - in der Telefon-, Mail- und Chatberatung - hat (für Onlineberatung siehe z.B. Gahleitner (2005)) und eine wichtige Ergänzung zu Face-to-Face-Kriseninterventionseinrichtungen bietet (Stein 2015).

Zusammenfassend ist festzustellen, dass die Bedeutung der Krisenbewältigungsstrategien durch den dialogischen Kommunikationsprozess, das vielseitig eingesetzte Kommunikationswerkzeug und durch die dabei gewonnenen Informationen positiv beeinflusst wird (Bundy und Pfarrer 2015; Coombs 2015; Hegner et al. 2016; Park 2017).

\section{Telefonseelsorge und Krisenintervention per Telefon}

Das äußerst vertrauliche Angebot der TS bietet für Menschen in Krisen und schwierigen Lebenssituationen eine erste Anlaufstelle. In der Telefonberatung beginnen Gespräche oft unmittelbar, teilweise direkt aus dem aktuellen Geschehen heraus ohne Einstieg über eine formale Begrüßung (Seidlitz und Theiss 2007). Weder Anrufende noch Beratende haben Vorinformationen über die Person am anderen Ende der Leitung. Darüber hinaus ist die Wahrnehmung auf den akustischen Kanal reduziert. Dies fördert Assoziationen zu Geräuschen im Hintergrund - dadurch gibt es einen komplexen Interpretationsspielraum mit einem breiten Spektrum an Deutungsmöglichkeiten (Seidlitz und Theiss 2007).

Ein weiteres Merkmal der Telefonberatung ist, dass die Anrufe jederzeit abgebrochen werden können. Anrufende haben immer die Möglichkeit, das Gespräch schnell und ohne Angabe von Gründen zu beenden. Ferner gibt es keine Fortsetzungen der Gespräche: Bei erneuten Anrufen wird das Gespräch wahrscheinlich von einer anderen Beraterin oder einem anderen Berater entgegengenommen (Seidlitz und Theiss 2007).
Das „helfende Gespräch“ basiert vor allem auf den folgenden sieben tragenden Säulen in der Beratung: (1) zuhören, (2) klären, (3) ermutigen, (4) mittragen, (5) begleiten, (6) unterstützen/fördern beim Treffen eigener Entscheidungen und (7) informieren über weitere Beratungsstellen und Selbsthilfegruppen (Schohe 2006). Eine regionale und wohnortnahe Vernetzung wird in der Versorgungsforschung sowohl bei physischen als auch bei psychischen Beeinträchtigungen und Betreuungsnotwendigkeiten als eines der wichtigsten Handlungsfelder gesehen (Amelung et al. 2021; Stummer et al. 2016).

Die offene und vorurteilsfreie Haltung der Berater_innen ermöglicht die Selbstoffenbarung der Anrufenden auch bei sehr schambesetzten Themen und kann bei der Klärung der weiteren Vorgehensweise unterstützend wirken. Aktives Zuhören, ein verständnisvoller, empathischer Umgang mit den Anrufenden sowie Interesse und Aufmerksamkeit tragen dabei am Telefon zum Aufbau einer guten Beziehung bei im Sinne einer beziehungsfördernden Grundhaltung (vertiefend dazu Stein (2015, S. 81)). Vor allem der Aspekt des gelungenen Beziehungsaufbaus bildet die Grundlage für die Arbeit der Berater_innen (für konkrete Fallbeispiele am Telefon siehe Seidlitz und Theiss (2007, S. $159 \mathrm{f}$. und S. $166 \mathrm{ff}$.)).

Im weiteren Gesprächsverlauf schätzen die Berater_innen die Gefährdung der Betroffenen ein, z. B. ob eine akute Selbstgefährdung vorliegt. Hier „(...) kann das niedrigschwellige unterstützende Gespräch sich darauf fokussieren, verfügbare oder erreichbare Ressourcen zu erörtern, die möglichst direkt dem Menschen in der Krise eine erste Entlastung (...) gewährleisten sollen“ (Sötemann 2018, S. 29). Ergänzend dazu kann das Erfragen früherer suizidaler Krisen sowie hilfreicher Strategien in solchen Krisen sinnvoll sein (Sötemann 2018). Nach anschließender Zusammenfassung wird ein gemeinsamer Problemlösungsprozess initiiert (im Sinne eines möglichst konkreten nächsten Schrittes). Dabei kann beispielsweise der Fokus auf der Förderung der Distanzierungsfähigkeit von Suizidgedanken und auf Sinnsensibilisierung oder auf einem gemeinsamen Abwägen einer möglichen Kontaktaufnahme mit Psychotherapeut_innen liegen.

\section{Telefonseelsorge und Krisenintervention online}

Studien aus Deutschland legen nahe, dass die Beratungsformen - Mail und Chat - (a) insbesondere bei schambehafteten und vielschichtigen Themen (Döring 2003) und (b) für jüngere Personen Vorteile bieten (Kupfer und Mayer 2019). Die Onlineberatung per E-Mail und Chat gilt auch als „ideales Medium für Menschen, die Schwierigkeiten im direkten Kontakt und im Umgang mit anderen Menschen haben“ (Knatz und Dodier 2003, S. 13).

Durch die virtuelle Kommunikation im Rahmen der Onlineberatung wird dem Ratsuchenden ein ho- 
Tab. 1 Nutzungsdaten der Telefon- und Onlineberatung 2020

\begin{tabular}{|c|c|c|}
\hline & Telefonberatung & Onlineberatung \\
\hline \multicolumn{3}{|l|}{ Geschlecht } \\
\hline Männer & $43.150(30 \%)$ & 1973 (30\%) \\
\hline Frauen & $87.650(60 \%)$ & 3545 (53\%) \\
\hline Unbekannt & $15.220(10 \%)$ & $1116(17 \%)$ \\
\hline \multicolumn{3}{|c|}{ Alter in Jahren } \\
\hline $0-19$ & $2306(2 \%)$ & $1542(23 \%)$ \\
\hline 20-39 & $19.941(14 \%)$ & 2195 (33\%) \\
\hline $40-59$ & $60.614(41 \%)$ & 766 (12\%) \\
\hline $60-79$ & $28.434(19 \%)$ & $143(2 \%)$ \\
\hline Über 80 & $5342(4 \%)$ & $13(<1 \%)$ \\
\hline Unbekannt & $29.383(20 \%)$ & $1975(30 \%)$ \\
\hline
\end{tabular}

Tab. 2 Absoluter und relativer Zuwachs in der Telefonund Onlineberatung im Jahresvergleich 2019/2020

\begin{tabular}{|l|l|l|l|}
\hline Gesamt (2019) & Telefon & Mail & Chat \\
\hline Gesamt (2020) & 132.739 & 3120 & 790 \\
\hline Zuwachs absolut & 146.020 & 3526 & 3108 \\
\hline Zuwachs relativ & 13.281 & 406 & 2318 \\
\hline A & $9 \%$ & $13 \%$ & $293 \%$ \\
\hline
\end{tabular}

Anmerkung. Das Angebot der Chatberatung wurde zeitlich und personell ab März 2020 österreichweit aufgestockt

hes $\mathrm{Ma}$ an Kontrolle in seiner Selbstdarstellung und Selbstoffenbarung gewährleistet. Außerdem wird durch den virtuellen Raum ein Experimentieren mit unterschiedlichen Persönlichkeitsanteilen ermöglicht (Knatz und Dodier 2003, S. 18). Beim Schreiben kann die eigene Problemsituation erst einmal überlegt, sortiert, reflektiert werden, zudem können Gefühle geäußert und Spannungen abgebaut werden (vgl. Knatz und Dodier 2003, S. 13). Im Zuge dessen wird ein Prozess in Gang gesetzt, der zu einer psychischen Entlastung führen und persönliche Ressourcen aktivieren kann (Reindl et al. 2012). Dem Internet wird dabei auch meist mehr Anonymität als dem Telefon beigemessen (Kupfer und Mayer 2019).

Seit 2019 gibt es die Sofortchatberatung der TS, für die keine Terminvereinbarung notwendig ist. Diese ist über die Website telefonseelsorge.at/chatberatung erreichbar und täglich zwischen 16 und $22 \mathrm{Uhr}$ geöffnet. Verfügbaren Berater_innen kann zunächst eine Kurznachricht über den Anlass der Kontaktaufnahme übermittelt oder direkt (ohne Registrierung) in den Chatberatungsprozess eingestiegen werden. Einen konkreten Einblick in die Sofortchatberatung soll ein anonymisiertes Beispiel über den Beginn eines Chatverlaufs (Originaltext) ermöglichen (s. Anhang).

Die Telefonseelsorge Salzburg bietet darüber hinaus mit der "kids-line-Chatberatung“ ein spezielles Angebot für Kinder und Jugendliche an. Anders als bei der „kids-line“ wird der Sofortchat der TS öster-
Tab. 3 Themen in der Telefon- und Onlineberatung (absolute und relative Zahlen 2020)

\begin{tabular}{llll}
\hline Themen & Telefonberatung & Onlineberatung \\
\hline Einsamkeit/solation/Alltagsbewältigung & $40.640(28 \%)$ & $775(12 \%)$ \\
\hline Körperliche Krankheit & $9905(7 \%)$ & $183(3 \%)$ \\
\hline Psychische Krankheit & $34.720(24 \%)$ & $988(15 \%)$ \\
\hline Sucht & $2916(2 \%)$ & $39(<1 \%)$ \\
\hline Suizid & $1420(1 \%)$ & $564(9 \%)$ \\
\hline Krisen & $4087(3 \%)$ & $1214(18 \%)$ \\
\hline Sinn-/Glaubensfragen & $2048(1 \%)$ & $61(1 \%)$ \\
\hline Beziehung & $23.307(16 \%)$ & $651(10 \%)$ \\
\hline Sexualität & $1410(1 \%)$ & $140(2 \%)$ \\
\hline Gewalt/Missbrauch & $1168(<1 \%)$ & $190(3 \%)$ \\
\hline Arbeitswelt/Schule & $2937(2 \%)$ & $218(3 \%)$ \\
\hline Materielle Probleme & $1982(1 \%)$ & $48(<1 \%)$ \\
\hline Information/Fachauskünfte & $4216(3 \%)$ & $282(4 \%)$ \\
\hline $\begin{array}{l}\text { Sonstiges } \\
\text { Stelleninterne Themen (Telefon)/Test-, }\end{array}$ & $11.426(8 \%)$ & $667(10 \%)$ \\
\hline Stör- und Scherzanfragen (Online) & $3838(3 \%)$ & $389(6 \%)$ \\
\hline Nicht zustande gekommene Chats & $0(0 \%)$ & $395(6 \%)$ \\
\hline $\begin{array}{l}\text { Anmerkung. Die Zahlen beziehen sich auf Österreich. Chat- und Mailbera- } \\
\text { tung bilden die Onlineberatung }\end{array}$ & & \\
\hline
\end{tabular}

reichweit vorwiegend von Jugendlichen und jungen Erwachsenen in Anspruch genommen.

Nach beinahe zehn Jahren der anonymen Onlineberatung (Mail und Chat) zeigt sich, dass dieses Angebot vor allem jüngere Personen anspricht, über $50 \%$ der Ratsuchenden sind unter 30 Jahre alt, in Tab. 1 finden sich die aktuellen Statistiken aus Österreich. Während der Sars-CoV2-Pandemie ergab und ergibt sich dabei ein weiterer Vorteil: In Zeiten von Homeschooling und Homeoffice hatten und haben einige Personen etwa nicht die Ruhe, um ungestört und vor allem ungehört zu telefonieren.

Tab. 2 zeigt den Anstieg der Nachfrage im Jahr 2020 im Vergleich zum Vorjahr 2019. Grundsätzlich ist ein Zuwachs bei allen Kommunikationsmedien zu beobachten. In Bezug auf die Chatberatung fällt dieser jedoch besonders ins Auge: Hier sind die Anfragen um fast $300 \%$ gestiegen. Dies wird durch eine erhöhte Nachfrage begründet, aber auch durch eine zeitliche und personelle Ausweitung des Angebotes ab Mitte März 2020, um diesem erhöhten Bedarf gerecht werden zu können.

Auffallend bei der Onlineberatung ist, wie aus deutschen Studien hervorgeht, der hohe Anteil an schambesetzten Themen (Götz 2009), wie z.B. selbstverletzendes Verhalten (Eichenberg 2011; Feikert 2016), suizidale Krisen (Störr 2013; Oswald 2018; Gekeler 2018; Culemann 2002), Missbrauch (Meßmer et al. 2012; Eichenberg und Malberg 2012) oder Suchtproblematik (Drda-Kühn et al. 2018; Maier und Schaub 2013). Dies deckt sich mit den Erfahrungen der Telefonseelsorge. Deutlich wird, dass das Thema Einsamkeit in der digitalen Kommunikation wesentlich weniger vorkommt. Wohingegen Themen wie Gewalt/Missbrauch, Suizid, 
Beziehungsprobleme und psychische Gesundheit allgemein wesentlich häufiger angesprochen werden. Das kann natürlich sowohl am Medium selbst als auch an den unterschiedlichen Altersgruppen der Mediennutzer_innen liegen (Tab. 3).

\section{Trends für die Zukunft}

Das Kommunikationsverhalten der einzelnen Generationen ändert sich stark. War vor einigen Jahren Facebook noch ein junges Medium, so sind es aktuell andere Dienste, insbesondere Messenger, die genutzt werden (Institut für Jugendkulturforschung 2021). Diese Veränderung wird auch in der niederschwelligen, kostenfreien Beratung ihre Spuren hinterlassen. Konkret konnte dies bereits durch eine deutschlandweite Studie über die Veränderung der Gesprächskultur aus dem Jahr 2010 bestätigt werden: Etwa $89 \%$ der über 60-Jährigen sahen die Möglichkeit eines guten Gesprächs lediglich via Face-to-Face, d.h. weder per Telefon noch per Internet. Dieser Wert sank bei den 14-17-Jährigen auf $44 \%$. In letzter Gruppe überwog mit $46 \%$ auch die Meinung, dass gute Gespräche auch über Messenger, Chat, Mail und andere internetbasierte Kommunikationsformen durchgeführt werden können und nichts verloren ginge (Institut für Demoskopie 2010). Seit dem Jahr 2010 ist eine Fortsetzung dieses Trends zu beobachten. In Österreich sind große Ähnlichkeiten zu erwarten, obwohl dazu keine repräsentativen Untersuchungen vorliegen.

Die Nutzung von Beratungsapps, Kurznachrichten oder auch Messenger im psychosozialen Bereich dürfte stark zunehmen. Dies stellt natürlich einerseits die Betriebsorganisation und andererseits die Ausbildung der Mitarbeiter_innen vor die Herausforderung, professionelle Beratungen in diesen Formaten anzubieten. In der Psychotherapie und der Sozialen Arbeit gibt es bereits in einigen Ländern Europas positive Erfahrungen mit Beratungsapps (Steinhart et al. 2014; Moessner und Bauer 2017). Dies würde aber dem TS-Charakter einer schnellen, barrierefreien und anonymen Zugänglichkeit widersprechen (z. B. entsteht möglicherweise durch das Herunterladen der App eine erste Hürde).

Die Telefonseelsorge setzt auf die Option sich ergänzender Kommunikationskanäle. So werden Klient_innen in akuten Krisen im Rahmen der Onlineberatung dazu motiviert, zusätzlich möglichst zeitnahe Kontakt mit dem Notrufdienst 142 der Telefonseelsorge aufzunehmen.

Hervorzuheben ist, dass über die Social-MediaKanäle wie Facebook, Instagram oder über den WhatsApp-Messenger keine Beratungen der TS stattfinden, da über diese Medien die Datensicherheit und die zwei wichtigsten Grundsätze der TS - nämlich „Vertraulichkeit“ und „Anonymität“ nicht gewährleistet werden können. Eine Erweiterung des Portfolios der Onlineberatungskanäle muss mit diesen Grundsätzen einhergehen, wobei laufende organisatorische Ad- aptierungen und eine entsprechende Flexibilität der Mitarbeiter_innen ebenso erforderlich sind. Darüber hinaus wird auch die Aneignung von Fachwissen über den Einfluss medialer und digitaler Entwicklungen als essenzieller Bestandteil von Fortbildungsveranstaltungen im Onlineberatungsbereich berücksichtigt (Engelhardt 2018).

Die Berater_innen können frei wählen, über welches Medium sie tätig sein wollen. Ein weiterer Aspekt ist die Erweiterung des Beratungsteams durch die gezielte Gewinnung von jüngeren Berater_innen, die eher die Nutzung digitaler Medien bevorzugen. Zur Gewinnung jüngerer Berater_innen läuft derzeit ein EU-Projekt („Chat your value“) unter der Projektleitung von IFOTES.

Derzeit gibt es noch kaum wissenschaftliche Literatur, die sich mit Beratung über Messenger auseinandersetzt. Einen kurzen Überblick gibt z. B. Engelhardt (2018, S. 66 f.). Die folgenden abschließenden Annahmen und Erwartungen erfolgen demnach erfahrungsbasiert. Es ist anzunehmen, dass der Einsatz von Messenger in der Beratung zu einer Veränderung der Kommunikation führt. Als vorteilhaft wird hierbei erachtet, dass der ganze Beratungsverlauf ständig sichtbar ist - dies kann Kohärenz und Kontinuität wiederum positiv beeinflussen. Dadurch wird die inhaltliche Kommunikationsebene beeinflusst bzw. wird der Beratungsprozess zeitlich ausgedehnt - vergleichbar einer Chatberatung, die sich über mehrere Tage erstrecken kann (Engelhardt 2018).

Ferner ist es wahrscheinlich, dass Ratsuchende vorwiegend von einem Smartphone schreiben. Das kann bedeuten, dass sich die „Schreiborte“ verändern, z. B. hin zu einer Anfrage von unterwegs, wie beispielsweise aus der U-Bahn. $\mathrm{Zu}$ überlegen ist daher, welche Adaptierung der Onlineberatungsangebote durch diese Veränderungen des Beratungssettings erforderlich sind, um einen solchen dialogischen Beratungsprozess adäquat und hilfreich anzubieten.

Zudem kann es zu einer vermehrten Auflösung regionaler Grenzen kommen - wie dies auch in verschiedenen Disziplinen unter dem Begriff Entgrenzung diskutiert wird (für eine ausführliche Definition des Begriffs Entgrenzung siehe z. B. Ebner-Zarl (2021, S. $1 \mathrm{ff}$.)). Für die optimale psychosoziale und regionale Versorgung erscheint es sinnvoll, verstärkt über Ländergrenzen hinweg vernetzt zusammenzuarbeiten, z. B. im gesamten deutschsprachigen Raum.

Die Sars-CoV2-Pandemie hat diese Entwicklung jedenfalls massiv verstärkt. So berichten etwa die deutschen Jugendämter, dass sie Kontakt mit ihren Klient_innen zu $99 \%$ über Telefon, zu $94 \%$ über internetbasierte Kanäle und nur mehr zu $64 \%$ Face-toFace anbieten (Mairhofer et al. 2020). Vieles davon wird auch nach der Pandemie bestehen bleiben - mit den bereits genannten Herausforderungen an Ausbildung, Organisation und Qualität der Kommunikation im Zuge der Krisenintervention durch die Telefonseelsorge. 
Denkbar ist, zukünftig weiterhin technische Trends im Beratungsfeld der Telefonseelsorge (Telefon-, Mailund Chatberatung) aufzugreifen und etwa analog zu Messenger wie WhatsApp, ein datensicheres Messengerberatungsangebot zu schaffen. Damit wäre es den Ratsuchenden flexibel möglich, das Beratungsmedium entsprechend zu ihrer Zeit und ihrem Anliegen bzw. Thema zu wählen. Dies könnte vor allem jüngere Erwachsene in dem Kommunikationsmedium abholen, in dem sie sich ohnehin bewegen - wie dies bereits mit der aktuellen Mail- und Chatberatung gelungen ist. In der Telefonseelsorge ist und bleibt das Herstellen eines guten Kontaktes, respektive der Aufbau einer Beziehung, wesentlicher Wirkfaktor (Stein 2015).

\section{Einhaltung ethischer Richtlinien}

Interessenkonflikt P. Schadenhofer, H. Stummer und L. M. Curth geben an, dass kein Interessenkonflikt besteht.

Ethische Standards Der im Anhang zitierte Originaltext über den Beginn eines Chatverlaufs wurde entsprechend der COPE (Committee on Publication Ethics)-Leitlinien wiedergegeben.

Open Access Dieser Artikel wird unter der Creative Commons Namensnennung 4.0 International Lizenz veröffentlicht, welche die Nutzung, Vervielfältigung, Bearbeitung, Verbreitung und Wiedergabe in jeglichem Medium und Format erlaubt, sofern Sie den/die ursprünglichen Autor(en) und die Quelle ordnungsgemäß nennen, einen Link zur Creative Commons Lizenz beifügen und angeben, ob Änderungen vorgenommen wurden.

Die in diesem Artikel enthaltenen Bilder und sonstiges Drittmaterial unterliegen ebenfalls der genannten Creative Commons Lizenz, sofern sich aus der Abbildungslegende nichts anderes ergibt. Sofern das betreffende Material nicht unter der genannten Creative Commons Lizenz steht und die betreffende Handlung nicht nach gesetzlichen Vorschriften erlaubt ist, ist für die oben aufgeführten Weiterverwendungen des Materials die Einwilligung des jeweiligen Rechteinhabers einzuholen.

Weitere Details zur Lizenz entnehmen Sie bitte der Lizenzinformation auf http://creativecommons.org/licenses/by/4. $0 /$ deed.de.

\section{Anhang}

Beginn eines Beratungsgesprächs im Chat (anonymisierter Originaltext)

Ein Chat kann bis zu 50 min dauern. Für einen kurzen Einblick in den Sofortchat und aufgrund der begrenzten Zeichenzahl haben wir uns entschieden, lediglich den Anfang des Chats mit Beginn des Beziehungsaufbaus darzustellen.

Beraterin: Hallo! Willkommen bei der Onlineberatung der Telefonseelsorge. Anna ist mein Name. Wie kann ich Ihnen helfen?
Ratsuchende: ich bin gerade total überfordert von meinen Gefühlen und weiß gerade nicht, wohin mit mir ... und habe auch noch nie so ein Angebot wahrgenommen

Beraterin: Schön, dass Sie hergefunden haben!

Beraterin: Gefühlschaos also ... hmmm

Beraterin: Möchten Sie mir die Situation ein bisschen beschreiben?

Ratsuchende: eigentlich ist in meinem leben von außen alles super und läuft richtig gut, ich bin 31, bin seit einem Jahr verheiratet mit meinem Freund, den ich seit 12 Jahren kenne.. ich bin selbstständig und auch da läuft es gerade sehr gut

Ratsuchende: ich habe letzte Woche bei einem Auftrag einen Kollegen kennengelernt und wir sind zweimal zusammen im Bett gelandet, obwohl ich das normalerweise nicht mache.. weil ich werte wie treue, loyalität etc. sehr hoch halte.. aber dennoch ist es passiert.. und ich habe es sogar genossen, habe kein schlechtes gewissen und bin seitdem total überfordert von meinen gefühlen

Ratsuchende: ich habe gerade niemandem, mit dem ich darüber sprechen kann. ich kann es auch alles gar nicht richtig beschreiben. ich fühle so „aus der welt“ genommen

Ratsuchende: ich fühle mich gerade von der welt mit allen ihren gefühlen absolut überfordert und weiß gar nicht wohin mit mir.. ich spüre so eine unglaubliche, alles übertreffende sehnsucht die mich erschlägt

Beraterin: hmm so als hätten Sie sich über sich selbst erschrocken

Beraterin: Klingt nach sehr intensiven Gefühlen, die Sie gerade durchleben

Ratsuchende: ja, vor allem fühle ich mich gerade so unglaublich allein damit

Ratsuchende: wie ein kompass, der nicht weiß in welche richtung er sich ausrichten soll, da das magentfeld nicht mehr stimmt

Beraterin: Ja das kann ich mir gut vorstellen. Klingt nach viel Wind von allen Seiten und Orientierungslosigkeit

Ratsuchende: ich habe auch öfters panickattaken und todesangst bzw. verlustangst (im sinne von: das leben geht viel zu schnell vorbei)

Ratsuchende: Hallo Anna 
Beraterin: Haben Sie diese Panikattacken schon länger?

\section{Ratsuchende: ja}

Ratsuchende: schon ca. 1 jahr sehr stark, immer wieder

Beraterin: sind sie in letzter Zeit stärker?

Ratsuchende: ja, sie sind stärker geworden, vor allem hatte ich sie immer nur nachts, jetzt treten sie auch manchmal tagsüber auf

Beraterin: Das stelle ich mir als einen sehr unangenehmen Zustand vor ... Mit so großer Angst konfrontiert zu sein. Besonders auch, wenn Sie Todesangst verspüren.

Ratsuchende: und da wusste ich dann, dass ich dringend mal mit jemandem sprechen muss, bevor ich „verrückt“ werde

Beraterin: Was haben Sie das Gefühl, „drückt“ im Moment am meisten? Beziehungsweise: Was erhoffen Sie sich von unserem Chat?

Durch gezieltes Nachfragen der aktuellen Situation, das Evaluieren verfügbarer Ressourcen sowie des eigenen Handlungsspielraumes und des Selbstgestaltungspotentials kam es im Chatgespräch zu einer ersten emotionalen Entlastung. Im weiteren Chatverlauf ist die Ratsuchende davon überzeugt, dass für sie eine weiterführende Psychotherapie sinnvoll sein könnte.

\section{Literatur}

Amelung, V., Ex, P., \& Legeganck, M. (2021). Braucht ein komplexererVersorgungsbedarfauchkomplexereVersorgungsstrukturen? - Herausforderungen der ambulanten Versorgung. Gesundheitswesen, 83(5), 345-348. https:// doi.org/10.1055/a-1441-3262.

Bundy, J., \& Pfarrer, M. D. (2015). A burden of responsibility: the role of social approval at the onset of a crisis. Academy of Management Review. https://doi.org/10.5465/amr.2013. 0027.

Bundy, J., Pfarrer, M.D., Short, C.E., \& Coombs, W.T. (2017). Crises and crisis management: integration, interpretation, and research development. Journal of Management. https://doi.org/10.1177/0149206316680030.

Caplan, G. (1964). Principles of preventive psychiatry. New York: Basic Books.

Coombs, W. T. (2015). Thevalue of communication duringa crisis: Insights from strategic communication research. Business Horizons. https://doi.org/10.1016/j.bushor.2014.10. 003.

Culemann, A. (2002). Chancen und Grenzen der Onlineberatung für junge Menschen. Wege zum Menschen, 54(1), $20-36$.

Cullberg, J. (1978). Krisen und Krisentherapie. Psychiatrische Praxis, 5, 25-34.
Döring, N. (2003). Sozialpsychologie des Internet. Die Bedeutung des Internet für Kommunikationsprozesse, Identitäten, soziale Beziehungen und Gruppen (2. Aufl.). Göttingen: Hogrefe.

Drda-Kühn, K., Hahner-Korinski, R., \& Schlenk, E. (2018). Mit Smartphone, Tablet und Sozialen Medien - OnlineBeratung und -Therapie für die Generation der „Digital Natives“. E-Beratungsjournal, 14(1), 28-37.

Ebner-Zarl, A. (2021). Die Entgrenzung von Kindheit in der Mediengesellschaft.:Springer.

Eichenberg, C. (2011). Psychotherapie und Internet. Psychotherapeut, 56(6), 468-474. https://doi.org/10.1007/ s00278-011-0865-9.

Eichenberg, C., \& Malberg, D. (2012). Sexualität und Internet. Psychotherapeut, 57(2), 177-190.

Engelhardt, E. M. (2018). Lehrbuch Onlineberatung (1. Aufl.). Göttingen:Vandenhoeck\& Ruprecht.

Feikert, L. (2016). Zugänge, Schnittstellen, Nutzen-Ergebnisse einer empirischen Analyse von den Online-Beratungsangeboten der bke-jugendberatung und von [U25]Deutschland. E-Beratungsjournal, 12(1), 14-33.

Gahleitner, S. B. (2005). Neue Bindungen wagen. Beziehungsorientierte Therapie bei sexueller Traumatisierung. Personzentrierte Beratung \&Therapie, Bd.2. München:Reinhardt.

Gekeler, C. (2018). Auf der sicheren Seite. Standards zum Datenschutz der bke-Onlineberatung. E-Beratungsjournal, 13(2), 75-82.

Götz, N. (2009). Aufgefangen im Netz. Sozial Extra, 33, 18-20.

Hegner, S. M., Beldad, A. D., \& Kraesgenberg, A. L. (2016). The impact of crisis response strategy, crisis type, and corporate social responsibility on post-crisis consumer trust and purchase intention. Corporate Reputation Review. https://doi.org/10.1057/s41299-016-0007-y.

Humer, E., Pieh, C., Probst, T., Kisler, I.-M., Schimböck, W., \& Schadenhofer, P. (2021). Telephone emergency service 142 (TelefonSeelsorge) during the COVID-19 pandemic: cross-sectional survey among counselors in Austria. International Journal of Environmental Research and Public Health, 18(5), 2228. https://doi.org/10.3390/ ijerph18052228.

IFOTES (2021). About. IFOTES. https://www.ifotes.org/en/ about.Zugegriffen: 17.Aug. 2021.

Institut für Demoskopie Allensbach (2010). Gesprächskultur 2.0:WiediedigitaleWeltunserKommunikationsverhalten verändert Ergebnisse einer bevölkerungsrepräsentativen Studie zur Nutzung und Bewertung von Online-Kommunikation. https://www.ifd-allensbach.de/fileadmin/ studien/7490_Gespraechskultur.pdf. Zugegriffen: 17 Aug 2021

Institut für Jugendkulturforschung/ Saferinternet.at. (2021). Jugend Internet Monitor. https://www.saferinternet.at/ services/jugend-internet-monitor/. Zugegriffen: 18 Aug 2021

James, E. H., Wooten, L. P., \& Dushek, K. (2011). Crisis management: informing a new leadership research agenda. The Academy of Management Annals, 5(1), 455-493. https:// doi.org/10.1080/19416520.2011.589594.

Knatz, B., \&Dodier, B. (2003). Hilfe aus dem Netz. Theorie und Praxis der Beratung per E-Mail (Leben lernen). München: Klett-Cotta.

Kupfer, A., \& Mayer, M. (2019). Digitalisierung der Beratung. Onlineberatung für Kinder und Jugendliche und die Frage nach Möglichkeiten des Blended Counseling in der Kinder- und Jugendhilfe. Soziale Passagen, 11(2), 243-265.

Madsen, P. M., \& Desai, V. (2010). Failing to learn? The effects of failure and success on organizational learning 
in the global orbital launch vehicle industry. Academy of Management Journal, 53(3), 451-476. https:// doi.org/10. 5465/amj.2010.51467631.

Maier, L. J., \& Schaub, M. P. (2013). Ist-Analyse webbasierter Beratungs- und Selbsthilfeangebote in der Schweiz. Begleitforschung eSuchtportal.: Schweizer Institut für Sucht und Gesundheitsforschung (ISGF).

Mairhofer, A., Peucker, C., Pluto, L., van Santen, E., \& Seckinger, M. (2020). Kinder- und Jugendhilfe in Zeiten der CoronaPandemie.DJI-Jugendhilfeb@rometerbei Jugendämtern. München: Deutsches Jugendinstitute.V.

Meßmer, S., Weinhardt, M., \& Bauer, P. (2012). Kindeswohlgefährdung und Onlineberatung - ein Fallbeispiel. E-Beratungsjournal, 8(1), 1-14.

Mikušová, M., \& Horváthová, P. (2019). Prepared for a crisis? Basic elements of crisis management in an organisation. Economic Research-Ekonomska Istrazivanja, 32(1), 1844-1868. https://doi.org/10.1080/1331677X. 2019.1640625.

Moessner, M., \& Bauer, S. (2017). E-Mental-Health und internetbasierte psychotherapie. Psychotherapeut, 62(3), 251-266.

Oswald, A. (2018). Onlineberatung-Ist Mailberatung noch eine angemessene Form, um Jugendliche und junge Erwachsene in (suizidalen) Krisen zu erreichen? Eine soziotechnischeAnalyse. E-Beratungsjournal, 14(1), 1-15.

Park, H. (2017). Exploring effective crisis response strategies. Public Relations Review. https://doi.org/10.1016/j. pubrev.2016.12.001.

du Plessis, C. (2018). Social media crisis communication: Enhancing a discourse of renewal through dialogic content. Public Relations Review. https://doi.org/10.1016/j. pubrev.2018.10.003.

Reindl, R., Hergenreider, M., \& Hünninger, J. (2012). Schriftlichkeit in virtuellen Beratungssettings. In H. Geißler \& M. Metz (Hrsg.), E-Coaching und Online-Beratung: Formate, Konzepte, Diskussionen (S. 339-357). Wiesbaden: VS.

Rek, I., \& Dinger, U. (2016). Who sits behind the telephone? Interpersonal characteristics of volunteer counselors in telephone emergencyservices. Journal of Counseling Psychology.https://doi.org/10.1037/ cou0000157.

Schohe, S. (2006). Das Konzept der Telefonseelsorge. In T. Weber (Hrsg.), Handbuch Telefonseelsorge (2. Aufl. S. 25-33). Göttingen:Weber, Traugott.

Seidlitz, H., \& Theiss, D. (2007). Ressourcenorientierte Telefonberatung. Culemborg:Van Duuren Media.

Sonnek, G., Nestor, K., Tomandl, G., \& Voracek, M. (2012). Krisenintervention und Suizidverhütung (2. Aufl.). Wien: UTB, Facultas.

Sötemann, C. H. (2018). Telefonische Beratung in Kriseninterventionen. Berlin Heidelberg: Springer.

Stein, C. (2015). Psychotherapeutische Krisenintervention. Tübingen: Psychotherapie-Verlag.

Steinhart, I., Wienberg, G., \& Koch, C. (2014). Es geht doch! Krankenhausersetzende psychiatrische Behandlung in Deutschland-Praxiserfahrungen und Finanzierung. Psychiatrische Praxis, 41(08), 454-457.

Störr, A. K. (2013). „Ihr seid meine letzte Hoffnung“ Die Wirkung und Nachhaltigkeit der Online-Beratung für junge Menschen am Beispiel [U25]-Freiburg. E-Beratungsjournal, 9(2), 1-12.

Stummer, H., Katzdobler, S., Hecker, A., \& Noehammer, E. (2016). Herausforderungen an eine zukünftige medizinische Gesundheitsversorgung im Alpenraum und die Chancen für den Gesundheitstoruismus. In CIPRA Österreich (Hrsg.), Die Alpenkonvention und die Region der niederösterreichischen Randalpen: Möglichkeiten der nachhaltigen Regionalentwicklung (S. 68-72). Wien: CIPRA.

Tagarev, T., \& Ratchev, V. (2020). A taxonomy of crisis management functions. Sustainability. https://doi.org/10.3390/ su12125147.

Wenzel, J. (2008). Vom Telefon zum Internet: Onlineberatung der Telefonseelsorge. E-Mental-Health. https://doi.org/ 10.1007/978-3-540-75736-8 8 .

Hinweis des Verlags Der Verlag bleibt in Hinblick auf geografische Zuordnungen und Gebietsbezeichnungen in veröffentlichten Karten und Institutsadressen neutral. 